\title{
NATURALEZAY CULTURA: UNA LECTURA ELEMENTAL DE ARRÁNCAME LA VIDA DE ÁNGELES MASTRETTA
}

Leon Bodevin*

$\mathrm{E}$ n una entrevista con Bárbara Mujica publicada en Américas, Ángeles Mastretta se refiere a la situación de la mujer durante un período de revolución o agitación sociopolítica y asegura que "A menudo revoluciones cambian muy poco. Pero durante el período revolucionario la gente se atreve a hacer cosas que en tiempos de paz no harían: gozan de gran libertad, y no se preocupan de quién se acuesta con quién, y si te casas o no, o si vives con alguien, si la unión está documentada o es ilegítima. Esta aseveración infiere que el estallido de una acción revolucionaria se convierte en un lapsus que altera la conducta social: se puede asumir que el individuo siente, ya sea con aprehensión o beneplácito, la redundancia del guión del escenario social y promueve la subversión del discurso que concierta la norma, despojando de su tutela a los protocolos que ha redactado la cultura que lo respalda. El individuo retorna a sí mismo y modifica su interacción con el medio. A un nivel más de acuerdo a sus prioridades y necesidades, parcialmente ignora el mandato de la cultura.

La subversión de normas de conducta que produce el período revolucionario tiene un efecto más drástico en la percepción social de la mujer y

* Murray State University. 
en su conducta, ya que recontextualiza la relación entre ambos sexos en una circunstancia que se acerca más al mandato de la naturaleza, de la cual la norma social tiende a mantenerla a una distancia mayor que la asignada al hombre. Esta nueva disposición temporal abre las posibilidades de una nueva síntesis, la cual puede modificar su circunstancia en una forma permanente. Aminora la desigualdad de que ha sido objeto.

En Arráncame la vida, Mastretta expone una perspectiva de esa confluencia de la cultura y la naturaleza que configura la relación mujer/hombre. La vida de Catalina alegoriza el tedioso proceso de síntesis cultural que ha ido contextualizando esta relación, y se transforma en un paradigma del doble sentido con que la sociedad ha administrado las sanciones de su trato con la mujer y ha autorizado la extensión de privilegios al hombre.

A la naturaleza no le llevó mucho tiempo para convencer al hombre en los inicios de su deambular terrestre del gran poder del fuero que ella poseía para intervenir en su vida. La solución más elemental y a la vez la más humana y política la propuso la posibilidad de una relación de acatamiento, o sumisión a los elementos, para así amortiguar los efectos de su embestida. La adoración de sus componentes más agresivos apareció como un compromiso que habría de complacerla al saberse reconocida en su autoridad y juridicción, y así éstos adquirieron facultades mágicas. Brindaron al individuo no sólo la solución de sus incógnitas cósmicas, sino le ofrecieron también una opción para el feliz desenlace de sus tribulaciones diarias. En Sueño y alucinación en el mundo maya y nahuatl, Mercedes de la Garza ejemplariza en una muestra de hibridez cultural la continua adhesión al orden natural de estos pueblos: la invocación de un chamán quiché antes de iniciar el proceso de adivinación del mal que afecta a su paciente:

¡Salud nuestro padre que estás en el cielo, y también nuestro padre Jesucristo que está en el Calvario, y también nuestras primeras abuelas y abuelos que vinieron ante los altares, los dueños de la varapunta, los dueños de la semilla de pito, los dueños de la adivinación por el maiz y por la sangre! Vengan aquí (p. 183). 
El texto de Arráncame la vida enlaza curiosamente los antecedentes, raíces, de sus personajes principales en torno a una actividad común, la cual destaca al vecindario de la naturaleza con una vigencia cultural: capta momentos de la vida de Andrés Ascencio, y del padre de Catalina, Marcos Guzmán, además de Eulalia, la primera mujer de Ascencio, y su padre Refugio Núñez; también de Pablo, el amigo y compañero de Catalina durante su primer embarazo. Todos ellos habían estado involucrados en la actividad de la ordeñanza y distribución de leche: "Mientras, trabajaban ordeñando vacas [Eulalia y Refugio] y repartían leche en una carreta conducida por Andrés y jalada por un caballo viejo" (p. 34), "Pablo repartía leche en una carretita tirada por mulas" (p. 32); "Me gustaba pensar [Catalina] que era domingo y que aún era posible subirse en el burro [de su padre] que ese día no cargaba leche" (p. 10). Aunque esta actividad resulta ser una constante en las raíces, antecedentes, del hombre en general, resalta el paralelismo en estos personajes cuya relación media la vida de Catalina, y a través de sus respectivas formas de relacionarse se perfila una perspectiva del desarrollo de la conciencia de la protagonista.

Así, cuando Andrés Ascencio reclama para sí, de su suegro, los derechos sobre su esposa, "Acuérdese don Marcos que ella ya no es una niña y que en esta mesa mando yo" (p. 17), su autoridad emanaba del control que el hombre había tomado de la vida, y por ende, de la sexualidad de la muchacha. Por su parte, la asignación de responsabilidades y deberes que hace Andrés durante el procedimiento de su matrimonio con Catalina confirma una adhesión a una circunstancia similar forjada por los mismos principios: "No m'ija, porque así no es la cosa. Yo te protejo a tí, no tú a mí. Tú pasas a ser de mi familia, pasas a ser mía" (p. 16). Catalina se transforma en una posesión que él apresa a una temprana edad: "Entonces él tenía más de treinta y yo menos de quince" (p. 9). Dadas las circunstancias, la ceremonia civil del matrimonio no se completa sin la ayuda del juez, quien explica a Catalina que tiene que decir que sí, en lugar de un sumiso bueno. La articulación del sí, o del bueno, no es promovida por su individualidad, proviene de ese espacio de aceptación silenciosa que la cultura ha forjado en su interior. Pero la ambigüedad de la percepción genérica del derecho que muestra el sistema queda ilustrada a través de la palabra de Andrés Ascencio: "Yo no tengo por qué disimular, yo soy un señor, tú eres una mujer, y las mujeres cuando andan de cabras locas queriéndose coger a todo el que le pone a temblar el ombligo se llaman putas" (p. 75).

Las amenidades y el cambio de circunstancias económicas que el matrimonio con Andrés significó para Catalina, se aparejaron con sus contrariedades: "Andrés se levantaba con la luz, dando órdenes como si yo fuera su regimiento (...) Andrés me tenía guardada como un juguete con el que 
platicaba de tonterías, al que se cogía tres veces por semana y hacía feliz con rascarle la espalda y llevar al zócalo los domingos" (p. 19; 28). Aún cuando la calidad de la forma de relacionarse de las partes sigue su curso convencional, el estado de conciencia de Catalina acusa una expansión:

Era la mamá de sus hijos, la dueña de su casa, su señora, su criada, su costumbre, su burla. Quién sabe que era yo, pero lo tenía que seguir siendo por más que a veces me quisiera ir a un país extranjero donde él no existiera, donde mi nombre no se pegara al suyo, donde la gente me odiara o buscara sin mezclarme con su afecto o desprecio por él. (p. 55)

La naturaleza como mediadora de la conducta humana encuentra su expresión más estridente en la manifestación de la sexualidad. Ésta tiende a desbordarse del marco de contención que le ha impuesto la cultura mientras su percepcion mítica se va disolviendo en forma extremadamente lenta. El silencio y el eufemismo observado en su trato han multiplicado las contradicciones de su entendimiento llegando al extremo de proponer una variada fuente de soluciones sin respaldo empírico, o considerado incluso la utópica simpleza de su disolución. Pautas propagadas por la falta de información que Mastretta esboza con ejemplos que toman lugar en los días iniciales de la relación de Catalina con Andrés: tras su primera experiencia sexual, éste le pregunta si acaso sintió, a lo que la muchacha le responde que sintió, pero que el final no lo entendió, asunto que le dió varios días de preocupación tratando de explicárselo a sí misma. Su búsqueda la llevó hasta una gitana que la familiarizó con la sensibilidad de su propia anatomía. La palabra de Catalina ilustra otro ejemplo contextualizado dentro del mismo orden: "Cuando llegué ya estaban ahí, Mónica llorando porque Pepa le había asegurado que si alguien le da un beso de lengua le hacía un niño" (p. 21).

Lo que podríamos llamar la simiente básica de un delineamiento de conducta para la mujer, construcción cultural que ha levantado el hombre en Occidente, se puede encontrar en el siguiente apartado bíblico: “A la mujer dijo [Jehová], multiplicaré en gran manera tus dolores y tus preñeces; con dolor parirás los hijos; y a tu marido será tu deseo, y él se enseñoreará de tí” (Gen.3:16). Tal como otras manifestaciones de la naturaleza, la energía sexual insatisfecha provocó inseguridad y ansiedad en el hombre, y éste puso su solución en boca 
de un orden divino para oficializar su autoridad de acuerdo a su voluntad e intereses. Se asignó un derecho de propiedad. El hombre se autoasignó una juridicción. Predicamento que el canal diseminador y penetrante del discurso religioso convirtió en código. La proyección social de la mujer quedó circunscrita dentro de este montaje cultural. Se transformó en el guión de su identidad, y en el libreto de su papel en el escenario social. Catalina en su discurso se encarga de subvertir esta circunstancia: "Eso fue lo único bueno que tuvo mi embarazo de Verania" (p. 32), al referirse a sus excursiones campestres con Pablo, esfuerzo que repite en: "Mis dos embarazos los pasé furiosa. Qué milagros de la vida ni qué la fregada" (p. 95). Un componente crítico en su discurso que se niega a aceptar un destino de acatamiento y pasividad, destino que Simone De Beauvoir capta como:

Una no nace, sino se convierte, en mujer. Ningún azar biológico, sicológico, o destino económico determina la figura que la individualidad femenina presenta en sociedad; es la realidad totalizante de la sociedad la que produce esta criatura, ser intermedio entre el macho y el eunuco, aquél que se suele describir como femenino. (p. 301)

Ante esta circunstancia, Catalina desea establecer que la mujer es una realidad diferente de la leyenda esquematizada redactada por la cultura, que es una conciencia individual, y que en asuntos personales ella puede tomar sus propias decisiones. Sugiere la necesidad de consolidar que su interioridad es conciencia, y que reclama una nueva síntesis social. Circunstancia histórica que Lemaitre describe como:

Los datos históricos van a ir engarzándose al plano subjetivo de la historia de Catalina, reflejando su paulatina concientización (y la dialéctica de su liberación) y también su educación política que corre paralela a su educación sentimental tejiendo así el complejo discurso de lo que fue, para una mujer singular, la vida en el México de los años 30 y 40. (p. 189) 
La reacción de rechazo de su circunstancia encuentra una salida modificando ahora su conducta. Su repudio lo materializa inicialmente Catalina con una intención de diluír, o de decolorar su calidad de madre, a la cual la había conducido su matrimonio.

Después de la tarde que vomité, resolví cerrar el capítulo del amor maternal. Se los dejé a Lucina (los niños). Que ella los bañara, los vistiera, oyera sus preguntas, los enseñara a rezar y a creer en algo, aunque fuera en la virgen de Guadalupe. De un día para otro dejé de pasar las tardes con ellos, dejé de pensar en qué merendarían y en cómo entretenerlos. Al principio los extrañé. Llevaba años de estar pegada a sus vidas, habían sido mi pasión, mi entretenimiento. (p. 66)

A medida que materializa su expansión de conciencia, discernimiento de su condición, identifica su propia sexualidad como un componente de su penuria, a la vez que la designa como instrumento de presión para atenuar, o controlar, su circunstancia. Decide subvertir su política sexual: "Me trepó el vestido y yo apreté las piernas... Pero yo seguí con las piernas cerradas, bien cerradas por primera vez" (p. 74). La insurrección sexual que Catalina concreta se convierte en el canal de su necesidad de liberación, en su arma. Lo que involucra un esfuerzo inicial por evaporar, o comenzar a borrar, las marcas que caracterizan su matrimonio.

La mitología griega nos relata que Teresias, en una temprana etapa de su niñez, observó a dos serpientes en el proceso de apareamiento. El castigo que el niño recibió de los dioses por haber observado algo que no le correspondía, fue la pérdida de su masculinidad, y su transformación en una niña. Era una visión prohibida. No fue hasta que al cabo de siete años, esta niña se volvió a encontrar con el espectáculo de las serpientes, y sólo entonces se revirtió el proceso - Teresias volvió a convertirse en un sujeto masculino. Pero al cabo de algún tiempo, tropezó con la desnudez de Athena mientras ésta tomaba un baño, acto que ella castigó cegando para siempre al hombre. Se advierte en las dos experiencias de Teresias la presencia del tabú protegiendo la integridad de un secreto. En este plano imaginativo se puede arrogar que Catalina había adquirido una conciencia que trascendía las limitaciones que la concepción convencional del matrimonio le asignaba, la cual ahora atribuía la calidad de 
redundante, carente de sentido, a la actividad sexual propia de su matrimonio. Reconoce en términos reales los componentes de su matrimonio y decide terminar con un acto que tiene la particularidad de representar la unión. Percibe el espejismo que su propia falta de información había aceptado como real. Se apresta a transformarse en individuo. Inicia la disolución de los signos de identidad de un matrimonio que ya no la representa. Comienza su rebelión en contra de un caudillo que controla sus gustos y preferencias. A un nivel más personal, este caudillo se interpone a su intimidad, es la barrera que amaga sus espectaciones:

Yo me había dejado encerrar sin darme cuenta, pero desde ese día me propuse la calle. Hasta bendije la pendeja Unión de Padres de Familia que durante un tiempo sería mi pretexto.

- Juan, enséñame a manejar - le dije al chofer.

- Señora, me mata el general - contestó.

- Le juro que nunca sabrá cómo aprendí. Pero enséñame.

- Ora pues - dijo (p. 111).

Aprender a manejar aflora como una necesidad de movilidad. La materialización de una conducta que toma en cuenta su propia idiosincrasia y que ahora se manifiesta con el mismo desenfado con que se había consolidado su opresión. Busca nuevos espacios. Adquiere una convicción del potencial que le ofrece su nuevo estado consciente. Percibe que puede tomar control de su vida. Su matrimonio ha estado regido por una noción que ordena, oprime, no comparte, y que sobresimplifica sus complejidades personales. Le ha asignado sus prioridades y predilecciones, tal como la sociedad ha determinado su propia identidad. De Beauvoir (1974) menciona algunos arquetipos a través de los cuales nuestra cultura ha aprendido a percibir a la mujer:

Símbolos propios de grupos [humanos] y modelos sociales son a menudo definidos por medio de antónimos pareados; la ambivalencia aparece como una calidad intrínsica de lo Eternamente Femenino. La sagrada madre tiene como correlativo a la madrastra, la joven angelical tiene [a su vez] a la virgen perversa; así se dirá a veces que madre equivale a muerte, y que cada virgen es tan sólo espíritu o carne asignada al demonio". (p. 287) 
Es lo que el hombre ha determinado que sea sin solicitar su opinión. De esta coyuntura, contradicción entre cultura y naturaleza, Catalina busca escape, De Beauvoir agrega:

"Para casi todos los poetas, la mujer encarna la naturaleza; pero para Breton ella no sólo expresa la naturaleza: la emana. Pero porque la naturaleza no habla un lenguaje común y sencillo, es necesario penetrar hasta sus secretos para obtener su verdad, lo que es uno con su belleza"(p. 266). Idealización que eventualmente De Beauvoir interpreta como: "[para Breton] la mujer no tiene otra vocación, sino el amor"(p. 268). Premisa que la hace derivar que: "Breton no habla de la mujer como un sujeto" (p. 268). Por su parte, Paul Claudel "exalta a la doncella, la sirvienta femenina, la devota que se entrega a Dios cuando se rinde al hombre" (p. 284). Pero para Stendhal, como la mujer está oprimida, y ajena a los propósitos del hombre, ella está libre para ser leal consigo misma: “ ...y porque aquellas cosas que se suponen de ser de importancia se encuentran fuera de su alcance, la mujer riesga menos que el hombre el peligro de perderse en ellas; la mujeres tienen una mayor opción de preservar su naturalidad, su candor; esa generosidad que Stendhal pone por sobre cualquier otro mérito" (p. 272).

Paralelo al desarrollo de Catalina, emerge un nuevo discurso y un nuevo espacio personal. Es la expresión de un deseo que ahora se condensa en la mujer que reclama la integración de su totalidad. Ávida de intimidad, su sensibilidad llama al bolero para delinear idealmente este espacio que la justifique. Un solaz para su ego. Canta y busca un puntal de apoyo en su letra: "Arráncame la vida, y si acaso te hiere el dolor ha de ser de no verme porque al fin tus ojos me los llevo yo," o, "Estoy aturdida, yo, yo que estaba tan tranquila disfrutando de la calma que nos deja ese amor que ya pasó.” Placer vicario y apología, forjados por un yo que ahora reclama la atención que durante años se le había negado. Evento que Cragnolino interpreta:

En Arráncame la vida, la utilización de los géneros populares, el melodrama, la novela rosa y el bolero, como textos de la cultura que esquematizan, reducen y terminan por disolver la complejidad de los conflictos poder/subordinación, masculino/femenino son "citados" para ser problematizados y subvertidos (p. 131). 
Catalina busca una legitimación de su condición a través del bolero, el cual disloca narrativas intertextuales que jerarquizan y oprimen, a la vez que su interioridad busca reafirmación y afición a lo suyo, un apego que lleve consigo la capacidad de entender y satisfacer espectaciones: "Desde que ví a Fernando Arizmendi me dieron ganas de meterme en una cama con él" (p. 75). Ilusión que se vió frustrada debido a la homosexualidad del galán. Pero ya nada detenía su arranque:

\begin{abstract}
Me volví infiel mucho antes de tocar a Carlos Vives. No tenía lugar para nada que no fuera él. Nunca quise así a Andrés, nunca pasé las horas tratando de recordar el exacto tamaño de sus manos ni deseando con todo el cuerpo siquiera verlo aparecer. Me daba vergüenza estar así por un hombre, ser tan feliz y volverme dichosa sin que dependiera para nada de mí. (p. 121)
\end{abstract}

Se puede decir que la individualidad aflora en arrebato; nace un deseo de lo que Jaspers llamaría elevarse a la existencia, trascender hacia una existencia similar a la suya: obtener un esclarecimiento existencial. Forja un nuevo orden en su circunstancia marital, a la que ahora ella observa con nueva perspectiva y fría distancia:

Sin decirlo me volví distinta. Le pedí a Andrés un Ferrari como el de Lilia. Me lo dio. Quise que me depositara dinero en una cuenta personal de cheques, suficiente dinero para mis cosas, las de los niños y las de la casa. Mandé abrir una puerta entre nuestra recámara y la de junto y me cambié pretextando que necesitaba espacio. A veces dormía con la puerta cerrada. Andrés nunca me pidió que la abriera. Cuando estaba abierta, él iba a dormir a mi cama. Con el tiempo parecíamos amigos otra vez (p. 201).

La relación ha sufrido un vuelco. El matrimonio ahora parece obedecer a un nuevo contrato ecuánime a sus partes que tiene poco de común con la ortodoxia del contrato original; cambio que se puede condensar en las siguientes 
palabras: Catalina ha ganado una conciencia con la cual el sistema no contaba; ha subvertido la posición que le asigna su cultura. Desde su lugar en el mundo de la voz pasiva en que le había correspondido vivir, construye su realidad en un plano activo, y de objeto se transforma en sujeto [Sí]. El atropello a su interioridad, con el cual le había tocado vivir, le dejó como legado una familiaridad con el ensañamiento, el que ahora comienza a emerger: "Como quieras - dije besándolo en la cabeza y tapándolo como si quisiera amortajarlo. Después fui de puntas hasta la puerta y lo dejé durmiendo" (p. 144, 145). O como se refleja en: "A veces despertaba temblando, suda y suda, seguro que él dormía con la misma boba que se casó" (147), lo que también se detecta en la siguiente cita: "Andrés" dije bajito, y fingí que sacaba de mi cinto un puñal" (p. 166). Su yo se resiste a caminar al sacrificio, o a transar en el compromiso. Hace suyas las armas del hombre, caudillo, y sus formas de relacionarse; necesita una liberación total, y en su defensa de la naturaleza encuentra una justificación de su conflicto con la cultura en el discernimiento de Marcuse (1974): "El sacrificio sistemático de la libido, su rígidamente impuesto desvío hacia actividades y expresiones de utilidad social es cultura" (p. 29). Todo lo que hay de naturaleza en Catalina se rebela en contra del orden que quiere contextualizar su vida; su individualidad se niega a vivir en las sombras. El arma la otorga la mujer de Atincingo, Carmela, cuyo esposo había sido muerto por órdenes de Andrés:

...ahí me traía esas hojas de limón negro para mi dolor de cabeza y para otros dolores. El té de esas hojas daba fuerzas pero hacía costumbre, y había que tenerle cuidado porque tomado todos los días curaba de momento pero a la larga mataba. Ella sabía de una señora en su pueblo que se murió nomás de tomarlo un mes seguido, aunque los doctores nunca creyeron que había sido por eso. Me las llevaba porque oyó en la boda que me dolía la cabeza, y por si se me ofrecía para otra cosa. (p. 215)

Catalina añadió distancia a su visión del decaimiento de Andrés mientras éste se aficionaba más y más al té de limón negro. Nos dice que se iba poniendo viejo, un día le dolía un pie y al otro una rodilla. Bebía sin tregua brandy de la tarde a la noche y té de limón negro durante toda la mañana. Agrega que no le apenó verlo perder fuerza, y que salía con Alonso como si hubieran sido novios y volvía a la casa de madrugada, y que durante semanas no abrió la puerta de su 
cuarto. Sólo a veces, como quien visita a su abuelo, tomaba té con Andrés en las mañanas, y acerca del día de su funeral, Catalina agrega: "Estaba sola, nadie me mandaba. Cuántas cosas haría, pensé bajo la lluvia a carcajadas, sentada en el suelo, jugando con la tierra húmeda que rodeaba la tumba de Andrés. Divertida con mi futuro, casi feliz" (p. 226). Su matrimonio con Andrés la había familiarizado con la muerte. Recogió la proposición con naturalidad. Con indiferencia se aseguró que Andrés se posesionase del arma. Todo lo que había de conciencia en ella permitió al opresor cumplir con su propia destrucción. Camille Paglia asevera que el sexo es el punto de convergencia entre el ser humano y la naturaleza. Ella lo llama una intersección: "Esta intersección es la misteriosa encrucijada de Hecate (Parséfona y Artemisa) donde todo retorna por la noche (...) Es un lugar insoportable, maldito a la vez que encantado" (p. 3). Confluencia que Concepción Gimeno de Flaquer en El problema feminista ${ }^{1}$ percibe como foco/objeto de abuso: "La esclavitud de un sexo es más dolorosa, más infamante que la de una raza" (p. 531), y también: "No podemos resignarnos las compatriotas de Teresa de Jesús a ser juguete de amor, bibelot social, domingo del hombre, cosa con vida o cuerpo sin alma" (p. 535). Catalina busca una total reivindicación, además de borrar toda huella de opresión, degradadora de su conciencia. Para lograr su realización hubo de llevar a cabo su propia insurrección. Las tácticas de su levantamiento las aprendió de su opresor. De tal manera, el arrepentimiento redunda. Ella sabe que todo tiene su término, que vivir como sujeto involucra tanto la renovación como la insurrección. Ahora camina hacia nuevos espacios que su individualidad le exije.

\section{RESUMEN}

Durante un período revolucionario la gente parece ignorar el guión que rige su conducta social. El individuo retorna a sí mismo. La cultura suspende el acoso que ha impuesto en la naturaleza de la mujer. Esta puede ser leal a su naturaleza humana. Para liberarse de su circunstancia represiva Catalina Guzmán necesita llevar a cabo su propia revolución con el objeto de ser honesta a la individualidad de su naturaleza. En este su esfuerzo, la misma naturaleza, la tierra, personificada en la mujer de Atincingo asiste a Catalina en su esfuerzo. Catalina recobra su individualidad.

1 Madrid: J. Bravo, 1903. También incluído en JAGOE, C.; BLANCO, A.; ENRÍQUEZ DE SALAMANCA, C. La mujer en los discursos de género. Barcelona: Icaria, 1998. Esta última obra se ha usado en este trabajo. 
BODEVIN, L. Naturaleza y cultura:...

Expresiones-claves: Período revolucionario, conducta social, mandato de la cultura, tribulaciones diarias, elevarse a la existencia.

\section{ABSTRACT}

During a revolutionary period people seem to ignore the script that rules society's social behaviour. The individual returns to him/her self. The culture stops the harassment that has been imposed on women and their nature. Women become free to be loyal to human nature. To attain a liberation of her oppressive circumstance, Catalina Guzmán needs to materialize her own revolution in order to be loyal to her individuality. Supportive to her effort, nature itself, personified by the woman from Atincingo, assists Catalina's pursuit. So, Catalina recovers the freedom to be honest and loyal to herself.

Key-words: revolutionary period, social behaviour, culture's mandate, daily tribulations, elevate himself to existence

\section{REFERENCIAS}

APTER-CRAGNOLINO, A. Jugando con el melodrama: género literario y mirada femenina en Arráncame la vida de Angeles Matretta. Confluencia, v. 11, n. 1, p. 126-133, 1995.

AURORA GONZÁLEZ, M. Innovación en la actual novela feminista mexicana: Domecq, Mastretta y Sechovich. In: VILLEGAS, J. (Ed.). International Association of Hispanists, Congreso (1992): Irvine 1992. Irvine: University of California, 1994. p. 220-227.

BATAILLE, G. Erotism: death and sensuality. San Francisco: City Lights Books, 1986.

BEAUVOIR, S. The Second Sex. New York: Random House, 1974.

GOLD, J. N. Arráncame la vida: textual complicity and the boundaries of rebellion. Chasqui: Revista de Literatura Latinoamericana, 17- 2, p. 35-40, Nov. 1988.

JAGOE, C.; BLANCO, A.; ENRÍQUEZ DE SALAMANCA, C. La mujer en los discursos de género. Barcelona: Icaria, 1998.

LEMAITRE, M. La historia oficial frente al discurso de la "ficción" femenina en Arráncame la vida de Angeles Mastretta. Revista Iberoamericana, 152-174 (1996):185197. 
MARCUSE, H. Eros and Civilization: a philosophical inquiry into Freud. Boston: Beacon Press, 1974.

MASTRETTA, A. Arráncame la vida. México: Cal y Arena, 1996.

MUJICA, B. Women in love and War. Américas, v. 49, n. 4, p. 36-43, 1997.

OROPESA, S. A. Popular Culture and Gender/Genre Construction in Mexican Bolero by Angeles Mastretta. In: FOSTER, D. W.; REIS, R. (Ed.) Bodies and Biases: Sexualities in Hispanic Cultures and Literatures. Minnesota: University of Minnesota Press, 1996.

PAGLIA, C. Sexual personae. Art and decadence from Nefertiti to Emily Dickinson. New York: Vintage Books, 1991.

PÉREZ, J. A. El arte narrativo de Angeles Mastretta en Arráncame la vida. Texto Crítico, v. 3, n. 4, p. 7-16, 1997. 